\title{
Design of Modbus Wireless Communication System Based on Remote
}

\section{Data Transmission}

\author{
Yingjuan $\mathrm{ZHAO}^{\mathrm{a}}$, Jingnan $\mathrm{MA}^{\mathrm{b}}$, Shaojuan LI,Jia LI \\ Science Institute, Air Force Engineering University, Xi'an 710051, P.R.China; \\ a39097721@qq.com,b52389425@qq.com
}

\begin{abstract}
Keywords: RS485,Modbus RTU protocol,433MHz wireless communication, configuration software Abstract. Industrial temperature、 flow and other sensor signals can be transmitted to the computer in the main control room by the RS485 bus, Modbus RTU communication protocol . Sometimes the data must be transmitted wirelessly, due to the difficulties of construction site wiring. In this paper, $433 \mathrm{MHz}$ wireless communication module is adopted to realize the transmission between the host and intelligent instrument. Professional configuration software is used to design user-oriented human-computer interface, achieving real-time display of temperature, flow and other data, EXCEL storage and report analysis.
\end{abstract}

\section{Introduction}

In many applications, field temperature, flow and pressure signals should be transmitted to the host computer in real time. Then the host computer carries on the analysis to the collected data, thus controls the actuating organization to make the corresponding action. RS-485 serial bus standard is widely adopted when the communication distance is several tens of meters to over one thousand meters $^{[1]}$, and the standard is designed for communication between the measuring instruments. Using the balance transmission and differential reception, RS-485 has the ability to restrain the common-mode interference.

But in many practical systems, the sensors and the host computer are not put together. The host computer is placed in the control room, and the sensors are installed in the work-field or in the environment which is relatively poor outdoor (such as underground mines, mountains, lakes, forest, etc.). The distance between the two is usually from tens of meters to several kilometers ${ }^{[2]}$. If wiring, we need to do the installation of cables and trenching construction. A lot of manpower and material resources are needed in the occasion of inconvenient wiring. In this case, it is more convenient to use wireless communication.

In this paper, $433 \mathrm{MHz}$ industrial wireless communication module is used instead of wired RS485 network directly, to achieve wireless networking. The wireless transmission distance can reach 300 meters to 3 kilometers ${ }^{[3]}$.

\section{The realization method of wireless Modbus communication}

First of all, the PT100 temperature sensor ${ }^{[4]}$ is used to collect the temperature data of the field, and the 8 channels analog signals are sent to the intelligent periodic inspecting instrument. After the inspection instrument, we can get the digital signals, which are easy to be addressed and transmitted. At the same time, the instantaneous flow and cumulative flow of the field can be measured through the electromagnetic flowmeter. Then, the data signals of the intelligent instrument and the electromagnetic flowmeter are transmitted to the $433 \mathrm{MHz}$ wireless communication module through 
RS485. After wireless transmission, wireless communication module in the main control room to receive real-time data on site. Through the bus, the host can achieve real-time data display, EXCEL storage and data analysis. The specific system diagram is shown in Fig. 1.

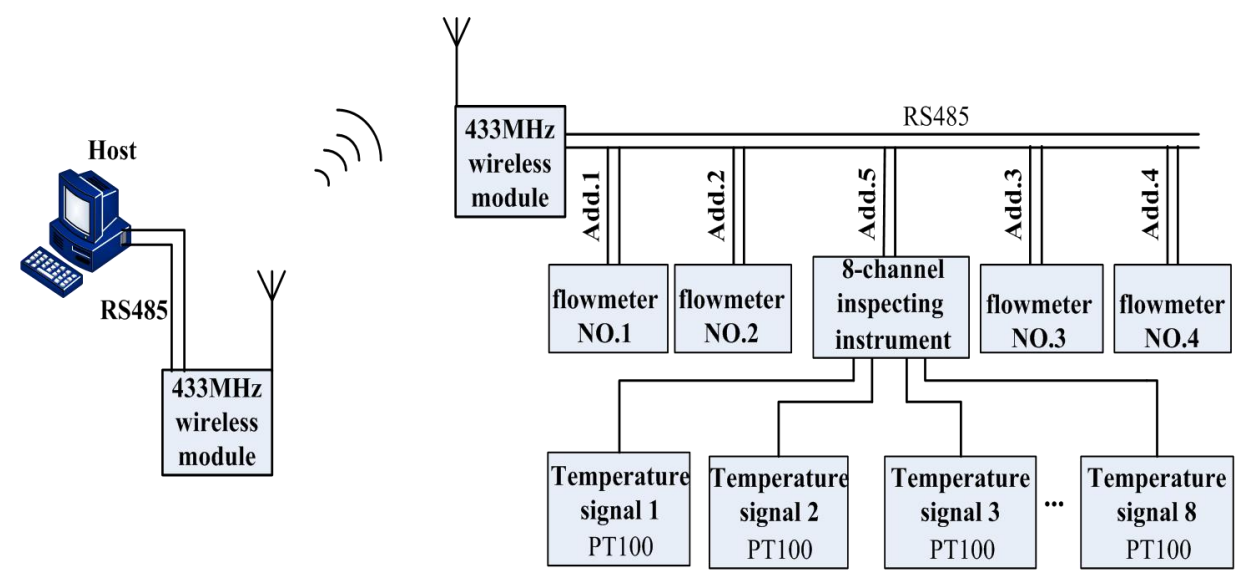

Figure1. Realization of wireless Modbus communication system

parameters setting of the intelligent instruments on site

In this paper, the intelligent instruments mainly include electromagnetic flowmeter, temperature sensor and 8-channel universal input inspecting instrument. The electromagnetic flowmeter has the standard MODBUS communication interface ${ }^{[5]}$,and its baud rate is $9600 \mathrm{bps}$. Set the serial port parameters of the electromagnetic flowmeter as follows: 1 bit start bit, 8 bits data bits, 1 stop bit, no parity bit. Then set the communication address of 4 flow meters respectively: Add.1, Add.2, Add.3 and Add.4, to ensure that each address is not repeated. 8 PT100 temperature sensors are respectively connected to eight-channel intelligent instrument.Set the communication address of the inspecting instrument to Add.5, and set the input data type of 8channels to 09. Due to the use of master-slave communication mode, the 8-channel data signals are transmitted to the host accurately only by Once addressing.

Through the wireless MODBUS communication network, the host can call the instrument according to the address, call the field data of the instrument at any time, such as the instantaneous flow, the instantaneous flow rate, the cumulative flow ,the temperature, and so on.

Setting of $433 \mathrm{MHz}$ wireless communication module

In this design, $433 \mathrm{MHz}$ wireless communication modules are chosen to send and receive data, so as to realize point to point communication between the host and the field equipments. The module uses the ISM open band $433 \mathrm{MHz}$, without the need to apply frequency point. The module's power supply is DC 9 24V (1A), and the average operating current is less than 50mA. The communication interface is based on the RS485 bus, Modbus RTU protocol. Its baud rate should be set to 9600bps, the serial port parameters should be set to 1 start bit, 8 data bits, 1 stop bit, and no parity bit.

The design of human-computer interface

The human-computer interface design software used in this paper is King-view 6.55. After creating the Kingview project, we need to create a new standard modbus device under the COM port of the device, and find the PLC-Modicon-modbus ${ }^{[6]}$ (RTU) in the list of the King-View device. (The electromagnetic flowmeter is driven by PLC-Modicon-modbus (RTU) ). Set address according to the address of the electromagnetic flowmeter, set the serial port parameters of COM, set the same baud rate with the electromagnetic flowmeter, both are $9600 \mathrm{bps}$, start bit, 8 data bits, 1 stop bit and no parity. Add the data variables of flowmeters in data dictionary. According to the description of King-view driver ,Modicon -modbus (RTU) variable name, register address and data format are shown in table 1 : 
Tab.1 variable name, register address and data format

\begin{tabular}{|c|c|c|c|c|}
\hline variable name & register address & data format & frequency & $\begin{array}{c}\text { read-write } \\
\text { property }\end{array}$ \\
\hline instantaneous flow & 34113 & Float & 500 & read-only \\
\hline instantaneous flow rate & 34115 & Float & 500 & read-only \\
\hline flow percentage & 34117 & Float & 500 & read-only \\
\hline fluid conductance ratio & 34119 & Float & 500 & read-only \\
\hline $\begin{array}{c}\text { integral part of Positive } \\
\text { cumulative value }\end{array}$ & 34121 & Long & 500 & read-only \\
\hline $\begin{array}{c}\text { decimal part of Positive } \\
\text { cumulative value }\end{array}$ & 34123 & Float & 500 & read-only \\
\hline $\begin{array}{c}\text { integral part of reverse } \\
\text { cumulative value }\end{array}$ & 34125 & Long & 500 & read-only \\
\hline $\begin{array}{c}\text { decimal part of reverse } \\
\text { cumulative value }\end{array}$ & 34127 & Float & 500 & read-only \\
\hline data conversion register & SwapL0 & Byte & 0 & write-only \\
\hline
\end{tabular}

After setting the above parameters, create a window interface and a data link, and then save the project and run the project. Serial port setting method of intelligent inspecting instrument in configuration software is the same with the electromagnetic flowmeter. The communication follows the RTU MODBUS protocol, and the instrument data format is: 2 byte register value $=$ high 8 bit binary data + low 8 bit binary data. Read register command format is shown in table 2, the starting address of the receiving register is from 40000, the specific data format is shown in table 3 . DE is device address - Add.5, CRC is the check byte. CRC -16 cyclic redundancy error check is adopted in this design. Register data is double byte, high in the front.

Tab. 2 read register command format

\begin{tabular}{|c|c|c|c|c|c|c|}
\hline 1 & 2 & 3 & 4 & 5 & 6 & $7 \sim 8$ \\
\hline $\mathrm{DE}$ & 3 & $\begin{array}{c}\text { starting register } \\
\text { high-byte }\end{array}$ & $\begin{array}{c}\text { starting register } \\
\text { low-byte }\end{array}$ & $\begin{array}{c}\text { register number } \\
\text { high-byte }\end{array}$ & $\begin{array}{c}\text { register number } \\
\text { low-byte }\end{array}$ & CRC \\
\hline
\end{tabular}

Tab. 3 receive register data format

\begin{tabular}{|c|c|c|c|c|c|c|c|}
\hline 1 & 2 & 3 & $4 \sim 5$ & $6 \sim 7$ & ---- & $\mathrm{M} * 2+2 \sim \mathrm{M}^{*} 2+3$ & $\mathrm{M} * 2+4 \sim \mathrm{M} * 2+5$ \\
\hline $\mathrm{DE}$ & 3 & $\begin{array}{c}\text { byte count } \\
\mathrm{M} 2\end{array}$ & $\begin{array}{c}\text { Register } \\
\text { data 1 }\end{array}$ & $\begin{array}{c}\text { Register } \\
\text { data 2 }\end{array}$ & & Register data M & CRC \\
\hline
\end{tabular}

The calculation method of fixed point number in configuration software is not unique, complement code can be adopted, anti-code can be adopted, too. Now we use the first channel sample value(in table 4) of 8-channel instrument as an example of floating-point algorithm to describe the principle of operation:05FE8CD1: 05 are integral bits ,FE8CD1 are decimal bits

Tab.4 the first channel sample value

\begin{tabular}{|c|c|c|c|c|c|c|c|}
\hline 0 & 0 & 0 & 0 & 0 & 1 & 0 & 1 \\
\hline $\begin{array}{c}\text { numeral } \\
\text { sign bit }\end{array}$ & $\begin{array}{c}\text { exponent } \\
\text { character } \\
\text { bit }\end{array}$ & \multicolumn{7}{c|}{ order code bit } \\
\hline
\end{tabular}

If the numeral sign and the exponent character are both equal to 0 , the value is a positive number. If the numeral sign and the exponent character are both equal to 1, the value is a negative number, 2 $\mathrm{n}$ is the order code, and its value is determined by order code. The above numerical value is $25=32$ (integer), and its decimal bits from low to high order are as follows: 


$$
\begin{aligned}
& 1 * 16 / 256=0.0625 ; \quad(13(\mathrm{D})+0.0625) * 16 / 256=0.81641 ; \quad(12(\mathrm{C})+0.81641) * 16 / 256=0.80103 ; \\
& (8+0.80103) \quad * 16 / 256=0.55006 ; \quad(14(\mathrm{E})+0.55006) \quad * 16 / 256=0.90938 ; \quad ;(15(\mathrm{~F})+0.90938) \\
& * 16 / 256=0.99434 ;
\end{aligned}
$$

Thus the real-time measurement value of the first channel is obtained: $0.99434 * 25=31.819$

The final design of the system interface is shown in Fig. 2.Through the address, the host can call the instruments on the spot and collect real-time data, such as instantaneous flow, instantaneous flow, cumulative flow parameters and temperature. The data can be saved or output as report forms.

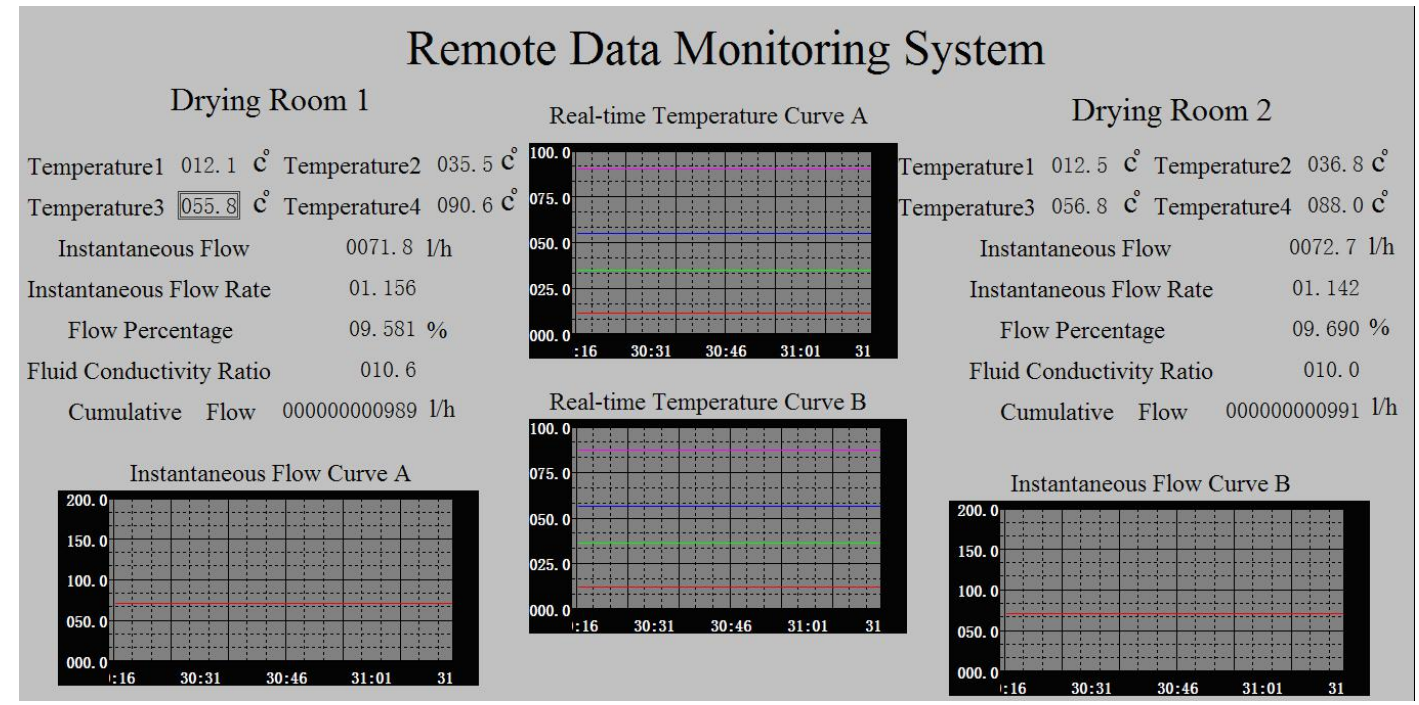

Figure2. design of the system interface

\section{Conclusions}

By testing, the Modbus wireless communication system ${ }^{[7]}$ based on intelligent instrument can running stably for a long time in the industrial field. The data collected from the main control room is completely in accord with data displayed on the screen of instruments on-site. The use of inspecting instrument greatly reduces the communication time, one time addressing can achieve multi-channel data acquisition. It can access a variety of sensors, such as pressure, liquid level sensors, etc. The system has good expansibility. For complex systems, programmable controller can also be added to the RS485, such as the popular SIEMENS, MITSUBISHI and other products. Wireless communication method is introduced, which greatly saves the cost, shortens the time limit for a project, reduces the wiring, so the system is also suitable for a variety of complex, harsh industrial environment.

\section{References}

[1] Design of Wireless Communication Scheme Between I/A Series and Interface System Based on Modbus Protocol[J]. Industrial Control Computer, 2012.

[2] Li H, Zhang H, Peng D. Design and Application of Communication Gateway of EPA and MODBUS on Electric Power System[J]. Energy Procedia, 2012, 17(Part A):286-292.

[3] Hall K H, Tutkovics S A, Vasko D A, et al. Extending industrial control system communications capabilities: US, US 20070186010 A1[P]. 2007.

[4] Guo Y, Du B X, Gao Y, et al. On-line monitoring system based on MODBUS for temperature measurement in smart grid[C]// Innovative Smart Grid Technologies - Asia. 2012:1-5.

[5] Zhao Y, Zhao H, Zhang Y, et al. Design of Gas Monitoring System Based on MODBUS and RS-485 Net Mode[J]. Computer Measurement \& Control, 2015. 
[6] Rzońca, D, Sadolewski, J, Stec, A, et al. Mini-DCS system programming in IEC 61131-3 structured text[J]. Journal of Automation Mobile Robotics $\backslash$ \&\&/sintelligent Systems, 2008.

[7] Kim H. 12. Wireless Communications Systems Design and Considerations[M]// Wireless Communications Systems Design. John Wiley \& Sons, Ltd, 2015:349-378. 